\title{
Surface of the Prostate
}

National Cancer Institute

\section{Source}

National Cancer Institute. Surface of the Prostate. NCI Thesaurus. Code C13091.

The external portion of the prostate including the anterior, inferolateral, lateral and posterior surfaces. 